\title{
PS2-15: Influence of atmospheric electric field over NM on CR intensity: Observations on Mt. Hermon and comparison with the theory
}

\author{
Lev Dorman ${ }^{1,2}$ \\ lid010529@gmail.com \\ Lev Pustil'nik ${ }^{1,3}$ \\ 3. Ariel University, Israel \\ levpust2149@gmail.com \\ Elizabeth Petrov ${ }^{4}$ \\ 4. Princeton University, NJ 08540, USA \\ elizabeth.petrov@princeton.edu
}

1. Israel Cosmic Ray \&Space Weather Center and Emilio Segre' Observatory, affiliated to Tel Aviv University, Shamir Research Institute, and Israel Space Agency, Israel;

2. Pushkov's IZMIRAN, Russian Academy of Science, Moscow, Russia;

1. Israel Cosmic Ray \&Space Weather Center and Emilio Segre' Observatory, affiliated to Tel Aviv University, Shamir Research Institute, and Israel Space Agency, Israel;

\begin{abstract}
On the basis of cosmic ray and atmospheric electric field one minute data obtained correspondingly by NM and EFS in Emilio Segre' Observatory (hight $2050 \mathrm{~m}$ above sea level, cut-off rigidity for vertical direction $10.8 \mathrm{GV}$ ) we determine the atmospheric electric field effect in CR for total neutron intensity and for multiplicities $m=1, m=2, m=3, m=4, m=5, m=6$, and $m=7$. For comparison and excluding primary CR variations we use also data obtained by NM of University "Roma Tre" (about sea level, cut-off rigidity 6.7 GV). According to the theoretical calculations of Dorman and Dorman (1995) the electric field effect in the NM counting rate must be caused mainly by catching of slow negative muons by lead nucleus with escaping few neutrons. As it was shown in Dorman et al. (1999), the biggest electric field effect is expected in the multiplicity $m=1$, much smaller in $m=2$ and negligible effect is expected in higher multiplicities. We will control this conclusion on the basis of our experimental data. Obtained results give a possibility to estimate total acceleration and deceleration of CR particles by the atmospheric electric field.
\end{abstract}

1. On the theory of atmospheric electric field effects in the hard muon component

1.1. Expected intensity of hard positive and negative muons

Because the atmospheric electric field effects are opposite for different charged muons, let us determine the expected intensity of positive and negative muons separately. According to the Dorman's theory of atmospheric variations of CR muon component without taking into account atmospheric electric field $(E(h)=0)$, the observed intensity of hard muons will be determined by the expression (Dorman, 1954, M1957, M1972)

$$
I_{\mu h}\left(h_{O}, E_{\mu \min }, Z\right)=I_{\mu h}^{+}\left(h_{O}, E_{\mu \min }, Z\right)+I_{\mu h}^{-}\left(h_{O}, E_{\mu \min }, Z\right)
$$

where $h_{o}$ is the atmospheric pressure on the level of observation, $\mathrm{Z}$ is the zenith angle, $E_{\mu \text { min }}$ is the apparatus muon energy threshold, and indexes + and - regard to positive and negative muons. Here

$$
I_{\mu h}^{ \pm}\left(h_{O}, E_{\mu \min }, Z\right)=\int_{0}^{h_{O}} d h_{2} \int_{E_{\pi}^{ \pm} \min }^{\infty} d E_{\pi} \int_{0}^{h_{2}} d h_{1} F^{ \pm}\left(E_{\pi}, h_{1}, h_{2}, h_{O}, Z\right)
$$

where $E_{\pi}$ is the energy of pions, $h_{1}$ is the level of pion generation, $h_{2}$ is the level of muon generation,

$$
E_{\pi \min }^{ \pm}=\left(E_{\mu \min }^{ \pm}+\int_{h_{1}}^{h_{O}} a^{ \pm}(h) d h\right)(\beta \cos Z)^{-1}
$$

is the lowest pion energy for producing positive and negative muons what can be detected by apparatus 
with threshold energy $E_{\mu \text { min }}$ (in the case of a plane-parallel screen). In Eq. 1.2 function

$$
\begin{aligned}
& F^{ \pm}\left(E_{\pi}, h_{1}, h_{2}, h_{0}, Z\right)=\frac{m_{\pi} c}{\tau_{\pi}} \frac{f_{\pi}^{ \pm}\left(E_{\pi}, h_{1}, Z\right)}{E_{\pi} \rho\left(h_{2}\right) \cos Z} \exp \left(-\frac{h_{2}-h_{1}}{l \cos Z}\right) \\
& \times \exp \left(-\frac{m_{\pi} c}{\tau_{\pi} E_{\pi} \cos Z} \int_{h_{1}}^{h_{2}} \frac{d h}{\rho(h)}\right) \exp \left(-\frac{m_{\mu} c}{\tau_{\mu}} \int_{h_{2}}^{h_{0}} \frac{d h}{\rho(h)}\left(\beta E_{\pi} \cos Z-\int_{h_{2}}^{h} a^{ \pm}(h) d h\right)^{-1}\right) .
\end{aligned}
$$

Here $m_{\pi}, m_{\mu}, \tau_{\pi}, \tau_{\mu}$ are the appropriate rest masses and life times of charged pions and muons, $\beta=m_{\mu} / m_{\pi}, \quad \rho(h) \quad$ is air density, $a^{ \pm}(h)$ is muon energy loss per $1 \mathrm{~g} / \mathrm{cm}^{2}$ (at $E(h)=0, a^{+}(h)=a^{-}(h) \approx 2 \mathrm{MeV} /\left(\mathrm{g} / \mathrm{cm}^{2}\right), f_{\pi}^{ \pm}\left(E_{\pi}, h_{1}, Z\right)$ is the pion generation function for positive and negative pions. Let us note that because primary CR out of the atmosphere are mostly positive particles (protons, $\alpha$-particles and heavies nucleus, there is a positive excess in the pion generation function: $f_{\pi}^{+}\left(E_{\pi}, h_{1}, Z\right)>f_{\pi}^{-}\left(E_{\pi}, h_{1}, Z\right)$. If $E(h) \neq 0$ then owing to acceleration or deceleration of charged muons their loses of energy will be change for positive and negative muons in opposite directions (decrease or increase correspondingly for accelerated or decelerated by electric field), and in this case $a^{+}(h) \neq a^{-}(h)$.

1.2. Expected influence of atmospheric electric field on intensity of hard positive and negative muons As mentioned in the previous section 1.1, if we take into account the atmospheric electric field, $a^{+}(h)$ and $a^{-}(h)$ for positive and negative muons become significantly different. For example, if $E(h)=+30 \mathrm{kV} / \mathrm{m}=+3 \times 10^{5} \mathrm{~V} /\left(\mathrm{g} / \mathrm{cm}^{2}\right)$ near sea level $\left(\rho(h) \approx 10^{-3} \mathrm{~g} / \mathrm{cm}^{3}\right)$, then for vertically arriving positive muons $a^{+}(h)=1.7 \mathrm{MeV} /\left(\mathrm{g} / \mathrm{cm}^{2}\right)$ and for negative muons $a^{-}(h)=2.3 \mathrm{MeV} /\left(\mathrm{g} / \mathrm{cm}^{2}\right)$ (in this case $\Delta a^{ \pm}(h)=\mp 0.3 \mathrm{MeV} /\left(\mathrm{g} / \mathrm{cm}^{2}\right)$ correspondingly for positive and negative muons). For the general case of muons arriving at zenith angle $\mathrm{Z}$ on the depth $\mathrm{h}$ with air density $\rho(h)$ one obtains

$$
\Delta a^{ \pm}(h)=\mp e E(h) \cos Z / \rho(h) .
$$

Using Eqs. 1.2-1.5, we obtain for atmospheric electric field effect in positive and negative muon intensity the following changes (caused by changes in muon energy looses according to Eq. 1.5):

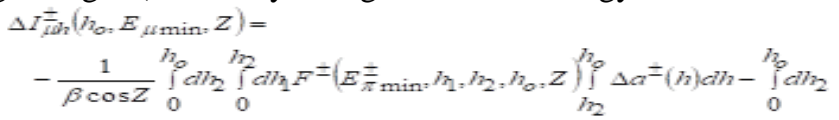

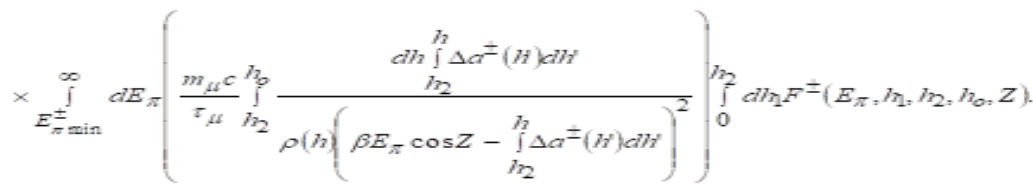

\subsection{Expected influence of atmospheric electric field on the total intensity of hard muons}

For the relative variation of the total intensity of hard muons caused by atmospheric electric field effect we obtain from Eq. 6 with taking into account Eq. 1.5:

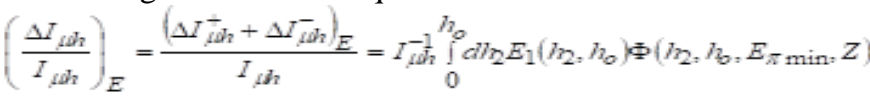

$$
\begin{aligned}
& +I_{\mu h h}^{-1} \int_{0}^{h_{0}} d h_{h_{2}} \int_{E_{n} \min }^{\infty} d E_{\pi} E_{2}\left(h_{2}, h_{o}, E_{\pi}, Z\right) \Phi\left(h_{2}, h_{o}, E_{\pi}, Z\right),
\end{aligned}
$$

where

$$
E_{1}\left(h_{2}, h_{O}\right)=\int_{h_{2}}^{h_{O}} \frac{e E(h) d h}{\beta \rho(h)} ; E_{2}\left(h_{2}, h_{O}, E_{\pi}, Z\right)=\frac{m_{\mu} c}{\tau_{\mu}} \int_{h_{2}}^{h_{O}} \frac{d h \int^{h_{O}} d h^{\prime}\left(e E\left(h^{\prime}\right) / \rho\left(h^{\prime}\right)\right)\left(\beta E_{\pi} \cos Z-a\left(h-h_{2}\right)\right)^{2}}{\rho\left(h_{2}\right.}
$$




$$
\Phi\left(h_{2}, h_{o}, E_{\pi}, Z\right)=\int_{0}^{h_{2}} d h_{1}\left(F^{+}\left(E_{\pi}, h_{1}, h_{2}, h_{o}, Z\right)-F^{-}\left(E_{\pi}, h_{1}, h_{2}, h_{o}, Z\right)\right) .
$$

\subsection{Absorption contribution to the atmospheric electric field effects in the total hard muon intensity}

The first integral in the right part of Eq. 1.6 and Eq. 1.7 describes the atmospheric electric field muon effect owed to changes of muon absorption. On the basis of calculations of integrals in Eq. 1.6 and Eq. 1.7 as made in Dorman (M1957, M1972), taking into account the difference between functions of positive and negative pion production in the Earth's atmosphere $f_{\pi}^{+}\left(E_{\pi}, h_{1}, Z\right)$ and $f_{\pi}^{-}\left(E_{\pi}, h_{1}, Z\right)$ we can roughly estimate the role of the absorption contribution in the muon atmospheric electric field effect. Let us assume that $E(h) \neq 0$ only in the region between $h_{O}$ and some level $h_{3}$, and have one sign. For $E(h)=10 \mathrm{kV} / \mathrm{m}$ and $30 \mathrm{kV} / \mathrm{m}, h_{o}=1000 \mathrm{~g} / \mathrm{cm}^{2}, h_{3}=700 \mathrm{~g} / \mathrm{cm}^{2}$ and $Z=0^{\circ}$ (vertical direction) we obtain the expected value of the effect at sea level $0.2 \%$ and $0.6 \%$ for hard muons (10 $\mathrm{cm} \mathrm{Pb}$ absorber). For Baksan station ( $h_{o} \approx 800 \mathrm{~g} / \mathrm{cm}^{2}$ ) at the same $h_{3} \approx 700 \mathrm{~g} / \mathrm{cm}^{2}$ we expect for $E(h)=10 \mathrm{kV} / \mathrm{m} 0.22 \%$ and for $30 \mathrm{kV} / \mathrm{m} 0.36 \%$ for threshold kinetic energies of muons $20 \mathrm{MeV}$ and $0.10 \%$ and $0.30 \%$ for threshold $90 \mathrm{MeV}$, in a good agreement with observations of Alexeenko et al. (1985, 1987).

\subsection{Decay contribution to the atmospheric electric field effects in total hard muon intensity}

The second integral in the right hand side of Eq. 1.6 and Eq. 1.7 describes the atmospheric electric field muon effect owed to changes of muon decay. For the same conditions that were considered in the previous Section 1.4 let us estimate the expected the decay contribution in the atmospheric electric field effects in the total intensity of hard muons on the sea level. The result of rough calculations is: $0.12 \%$ and $0.36 \%$ correspondingly for $E(h)=10 \mathrm{kV} / \mathrm{m}$ and $30 \mathrm{kV} / \mathrm{m}$. For Baksan station we estimate $0.23 \%$ and $0.69 \%$ for $20 \mathrm{MeV}$ threshold energy and $0.19 \%$ and $0.57 \%$ for $90 \mathrm{MeV}$ threshold energy, correspondingly for $E(h)=10 \mathrm{kV} / \mathrm{m}$ and $30 \mathrm{kV} / \mathrm{m}$. These values do not contradict those observed by Alexeenko et al. (1985, 1987).

\section{The short theory of atmospheric electric field effects in soft muon intensity}

Atmospheric electric field effects of soft muons (muons with small energy, absorbed by $10 \mathrm{~cm} \mathrm{~Pb}$ ) are especially interesting because they contribute in some part of the neutron monitor's and supermonitor's counting rates. It was shown in Dorman (M1957) that this leads to the temperature effect in the neutron component. As it was shown in Dorman and Dorman (1995), this will lead also to the appearance of atmospheric electric field effects in the neutron monitor's and super-monitor's counting rates.

2.1. General expression for expected intensity of positive and negative soft muons in an atmospheric electric field

The intensities of positive and negative soft muons will be determined by the expression

$$
I_{\mu s}^{ \pm}\left(h_{O}, R_{C}, Z\right)=\int_{0}^{h_{o}} d h_{2} \int_{E_{\pi 1}^{ \pm}}^{E_{\pi \min }^{ \pm} d E_{\pi}} \int_{0}^{h_{2}} d h_{1} F^{ \pm}\left(E_{\pi}, h_{1}, h_{2}, h_{o}, Z\right),
$$

where $F^{ \pm}\left(E_{\pi}, h_{1}, h_{2}, h_{O}, Z\right)$ was determined by Eq. 1.4 (see previous Section 1 ), and

$$
E_{\pi 1}^{ \pm}=\left(m_{\mu} c^{2}+\int_{h_{1}}^{h_{O}} a^{ \pm}(h) d h\right) / \beta \operatorname{cosZ} ; \quad E_{\pi \min }^{ \pm}=\left(E_{\mu \min }+\int_{h_{1}}^{h_{O}} a^{ \pm}(h) d h\right) / \beta \operatorname{cosZ} .
$$

In Eq. 2.2 the energy losses or increasing at the existing of atmospheric electric field $E(h)$ will be

$$
a^{ \pm}(h)=a+\Delta a^{ \pm}(h)=a \pm e E(h) / \rho(h)
$$

\subsection{Expected variations of positive and negative soft muon intensity in an atmospheric electric field} On the basis of Eq. 2.1 and Eq. 2.2 we obtain for variations of positive and negative soft muons: 


$$
\begin{aligned}
& \left(\Delta I_{\mu \omega}^{ \pm}\right)_{E}=\frac{1}{\beta \cos Z} \int_{0}^{h_{o}} d l_{2} \int_{0}^{h_{2}} d h_{1} \\
& \times\left(F^{ \pm}\left(E_{\pi 1}^{ \pm}, h_{1}, h_{2}, h_{0}, Z\right)-F^{ \pm}\left(E_{\pi}^{ \pm} \min , h_{1}, h_{2}, h_{o}, Z\right)\right) \int_{h_{2}}^{h_{b}} \Delta a^{ \pm}(h) d h_{1}-\frac{m_{\mu \mu} c}{\tau_{\mu}} \int_{0}^{h_{o}} d h_{2}
\end{aligned}
$$

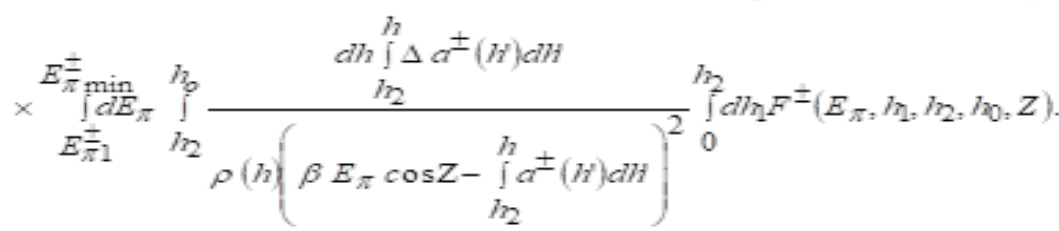

By taking into account Eq. 1.5 or Eq. 2.3 (where e is the charge of muons, and $E(h)$ is the vertical component of atmospheric electric field, positive in direction to the ground), we obtain from Eq. 2.4 for the relative change of positive and negative soft muon intensity caused only by atmospheric electric field effect the following expressions:

$$
\begin{aligned}
& \left(\Delta I_{\mu L}^{ \pm} / I_{\mu L}^{ \pm}\right)_{E}=\left(I_{\mu L}^{ \pm}\right)_{0}^{-1} \int_{0}^{h_{b}} d l_{2} E_{1}\left(h_{2}, h_{b}\right)\left(\Phi^{ \pm}\left(h_{2}, h_{b}, E_{\pi \min }, Z\right)-\Phi^{ \pm}\left(h_{2}, h_{b}, E_{\pi 1}, Z\right)\right) \\
& +\left(I_{\mu v}^{ \pm} \int_{0}^{-1} \int_{E_{\pi 1}}^{h_{b}} d h_{\pi \min } \int_{\pi} d E_{\pi} E_{2}\left(h_{2}, h_{b}, E_{\pi}, Z\right) \Phi^{ \pm}\left(h_{2}, h_{b}, E_{\pi}, Z\right),\right.
\end{aligned}
$$

where

$$
\begin{gathered}
E_{1}\left(h_{2}, h_{o}\right)=\int_{h_{2}}^{h_{o}} \frac{e E(h) d h}{\beta \rho(h)} ; \quad E_{2}\left(h_{2}, h_{o}, E_{\pi}, Z\right)=\frac{m_{\mu} c}{\tau_{\mu}} \int_{h_{2}}^{h_{o}} \frac{d h \int_{h_{2}}^{h} d h^{\prime}\left(e E\left(h^{\prime}\right) / \rho\left(h^{\prime}\right)\right)}{\rho\left(\beta E_{\pi} \cos Z-a\left(h-h_{2}\right)\right)^{2}} ; \\
\Phi^{ \pm}\left(h_{2}, h_{o}, E_{\pi}, Z\right)=\int_{0}^{h_{2}} d h_{1} F^{ \pm}\left(E_{\pi}, h_{1}, h_{2}, h_{o}, Z\right) .
\end{gathered}
$$

2.3. Absorption part of atmospheric electric field influence on soft positive and negative muon intensity

The first term in Eq. 2.5 describes the atmospheric electric field effect caused by absorption. According to Eq. 2.6 and Eq. 2.7 we obtain approximately:

$$
\left(\Delta I_{\mu \mathrm{S}}^{ \pm} / I_{\mu \mathrm{s}}^{ \pm}\right)_{E, a b s} \approx \pm E_{1}\left(h_{3}, h_{O}\right)\left(1-b^{\gamma+1}\right)\left(\left(m_{\mu} c^{2}+a\left(h_{o}-L\right)\right)\left(1-b^{\gamma}\right)\right)^{-1},
$$

where $L \approx 120 \mathrm{~g} / \mathrm{cm}^{2}$ is the attenuation path of primary CR in the atmosphere, $b=E_{\pi 1} / E_{\pi \min }$ and $\gamma$ is the power integral pion spectrum index for high energy region $(\geq 2 \mathrm{GeV}$ ). Let us suppose that $\mathrm{E}(\mathrm{h})=$ $10 \mathrm{kV} / \mathrm{m}$ and $30 \mathrm{kV} / \mathrm{m}$ between $h_{3}=700 \mathrm{~g} / \mathrm{cm}^{2}$ and $h_{o}=1000 \mathrm{~g} / \mathrm{cm}^{2}$. In this case we expect for soft positive and negative muons an effect of about $2.8 \%$ and $8.4 \%$, respectively.

\subsection{Decay part of atmospheric electric field influence on soft positive and negative muon intensity}

The second term in Eq. 2.5 describes the atmospheric electric field effect caused by the influence of acceleration and deceleration processes of muons on their decay. For this part we obtain approximately

$$
\left(\Delta I_{\mu \mathrm{s}}^{ \pm} / I_{\mu \mathrm{s}}^{ \pm}\right)_{E \text {, decay }} \approx \pm \frac{K c m_{\mu} \operatorname{cosZ}}{\rho\left(h_{4}\right) \tau_{\mu}}\left(h_{o}-h_{3}\right)\left(a\left(h_{o}-h_{4}\right)\right)^{-2} E_{1}\left(h_{3}, h_{4}\right),
$$

where $h_{3} \leq h_{4} \leq h_{o}$, the function $E_{1}\left(h_{3}, h_{4}\right)$ was determined by Eq. 2.6, and

$$
K=\left(1-\frac{(\gamma+1)\left(E_{\mu \min }+m_{\mu} c^{2}\right)}{a\left(h_{0}-L\right)}\right) /\left(\left(1+\frac{m_{\mu} c^{2}}{a\left(h_{0}-h_{4}\right)}\right)\left(1+\frac{E_{\mu \min }}{a\left(h_{0}-h_{4}\right)}\right)\right) .
$$

Using the same assumptions as in Section 2.3, we obtain for the expected amplitude of decay part of AEF effect $1.1 \%$ and $3.3 \%$ for $\mathrm{E}(\mathrm{h})=10$ and $30 \mathrm{kV} / \mathrm{m}$ between $h_{3}=700 \mathrm{~g} / \mathrm{cm}^{2}$ and $h_{o}=1000 \mathrm{~g} / \mathrm{cm}^{2}$. 


\section{Expected atmospheric electric field effects in neutron monitor total counting rate and in different multiplicities}

\subsection{Possible atmospheric electric field effects in neutron monitor}

In Dorman \& Dorman (1995), Dorman et al. (1995), the model of atmospheric electric field influence on CR was extended on the case of neutron monitor and super-monitor for total intensity, and in Dorman \& Dorman (1999) also for different multiplicities. It was taken into account that some part of the neutron monitor counting rate caused by negative soft muons captured by lead nucleons and formed mesoatoms with generation of neutrons of several $\mathrm{MeV}$ energy from lead. In this case, the neutron monitor or neutron super-monitor works as analyzer, which detect muons of only one, negative sign. It is very important because the atmospheric electric field effect has opposite signs for positive and negative muons that the main part of this effect in the muon telescope or in ionization chamber is compensated. The counting rate $I_{m}\left(h_{O}, R_{C}\right)$ of neutron monitor total intensity $(m=t)$ or different multiplicities $\mathrm{m}=1$, $2,3, \ldots$ at the pressure level $h_{O}$ and at the point with cut off rigidity $R_{C}$, is determined by

$$
I_{m}\left(h_{O}, R_{C}\right)=I_{m N}\left(h_{O}, R_{C}\right)+I_{m \mu s}^{-}\left(h_{O}, R_{C}\right),
$$

where the first term on the right hand of Eq. 3.1 $I_{m N}\left(h_{O}, R_{C}\right)$ reflects the main, nucleonic part of the neutron monitor counting rate. This part describes the generation of neutrons in the high atmosphere and their transport down to the observation level by scattering and absorption processes (the transport path for absorption is $\approx 145 \mathrm{~g} / \mathrm{cm}^{2}$; the atmospheric electric field has practically no influence on this part). The second term on the right hand of Eq. $3.1 I_{m \mu s}^{-}\left(h_{O}, R_{C}\right)$ reflects a very small part of the neutron monitor counting rate (e.g., for the total neutron intensity at sea level $I_{t \mu s}^{-}\left(h_{O}, R_{C}\right) / I_{t}\left(h_{O}, R_{C}\right) \approx 0.07$ ), caused by soft negative muons, producing lead mesoatoms with generation of neutrons in the detector.

Let us denote

$$
I_{m \mu s}^{-}\left(h_{O}, R_{C}\right) / I_{m}\left(h_{O}, R_{C}\right)=b_{m}\left(h_{O}, R_{C}\right) .
$$

Then the relative variation in neutron monitor counting rate due to the atmospheric electric field effect will be

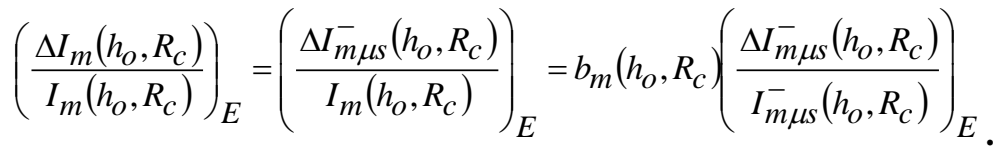

\subsection{Formation of lead mesoatoms in neutron monitor by soft negative muons}

The atmospheric electric field effect is determined mostly by soft negative muons generating lead mesoatoms with ejection of neutrons. Let us consider data on the frequency of lead mesoatoms formation in neutron monitors as a function of multiplicity and altitude. According to Nobles et al. (1967), special measurements were made in the region of middle cut off rigidity ( $R_{C} \approx 4-5 \mathrm{GV}$ ) and in the period of low solar activity (near 1965). It was found that the relative part of counting rate caused by formation of lead mesoatoms on the sea level is

$$
\left.b_{m}\left(h_{o}, R_{c} \leq 5 \mathrm{GV}\right)\right|_{\text {low SA }} ^{\text {s.l. }}=8.94 \% \text { for } m=1 ; 6.7 \% \text { for } m=2 ; 2.6 \% \text { for } m=3
$$

For the mountain level (about $3 \mathrm{~km}$ ) the relative part of counting rate caused by formation of lead mesoatoms is several times smaller:

$$
\left.b_{m}\left(h_{o}, R_{c} \leq 5 \mathrm{GV}\right)\right|_{\text {low SA }} ^{\mathrm{m} . \mathrm{l} \text {. }}=1.65 \% \text { for } m=1 ; 0.68 \% \text { for } m=2 ; 0.3 \% \text { for } m=3 \text {. }
$$

According to these data the largest atmospheric electric field effect at low solar activity is expected at sea level for multiplicities $m=1$ and $m=2$. At the mountain level the atmospheric electric field effect is expected to be about 5 times smaller (it is caused by much faster increase with increasing of altitude of the secondary neutron intensity from primary CR in comparison with the secondary muon intensity).

3.3. Dependence of lead mesoatoms formation in neutron monitor on cut off rigidity and solar activity

The relative part of neutron monitor counting rate caused by formation of lead mesoatoms is directly proportional to muon component intensity and inversely proportional to nucleonic component intensity. 
The muon component intensity decreases from $R_{C} \leq 2 \mathrm{GV}$ to $R_{c} \approx 15 \mathrm{GV}$ only by about $10 \%$, but intensity of neutron component decreases by about $50 \%$. This means that the relative part of neutron monitor counting rate caused by the formation of lead mesoatoms is expected to increase by about $40 \%$ from $R_{C} \leq 2 \mathrm{GV}$ to $R_{C} \approx 15 \mathrm{GV}$, and that on sea level this part will be increase and as result we expect:

$$
\left.b_{m}\left(h_{o}, R_{C} \approx 15 \mathrm{GV}\right)\right|_{\text {low SA }} ^{\text {s.l. }}=12.5 \% \text { for } m=1 ; 9.4 \% \text { for } m=2 ; 3.6 \% \text { for } m=3
$$

From the minimum to the maximum of solar activity at $R_{C} \leq 5 \mathrm{GV}$ intensity of muon component decreases by about $6 \%$ and neutron component by about $20 \%$; it means that the relative part of neutron monitor counting rate caused by formation of lead mesoatoms expected to be decrease by about $14 \%$ from minimum to maximum of solar activity, and as result we expect:

$$
\left.b_{m}\left(h_{o}, R_{c} \leq 5 \mathrm{GV}\right)\right|_{\text {high SA }} ^{\text {s.l. }}=10.2 \% \text { for } m=1 ; 7.6 \% \text { for } m=2 ; 3.0 \% \text { for } m=3
$$

Near equator ( $R_{C} \approx 15 \mathrm{GV}$ ) from the minimum to the maximum of solar activity intensity of muon component decreases by about $4 \%$ and intensity of neutron component by about $12 \%$; it means that the relative part of neutron monitor counting rate caused by formation of lead mesoatoms expected to be increase by about $8 \%$ from minimum to maximum of solar activity, and as result we expect:

$$
b_{m}\left(h_{o}, R_{C} \approx 15 \mathrm{GV}\right)_{\text {high SA }}^{\text {s.l. }}=13.5 \% \text { for } m=1 ; 10.2 \% \text { for } m=2 ; 3.9 \% \text { for } m=3
$$

\subsection{Atmospheric Electric Field (AEF) coefficients for NM on Mt. Hermon}

On the basis of results obtained in Section 3.3 we can determine atmospheric electric field coefficients $\mathrm{W}_{\mathrm{nE}}$ for observations by neutron monitor of the Emilio Segre' Observatory on Mt. Hermon:

$$
\begin{gathered}
\left.W_{n E}\left(h_{3} h_{o}, R_{c}=10.8 \mathrm{GV}, m=\operatorname{tot} ; 1 ; 2 ; 3\right)\right|_{10 \mathrm{SA}} ^{\mathrm{Mt} \mathrm{Hermon}} \\
\approx(4.37 ; 6.48 ; 4.32 ; 1.71) \times 10^{-5} \%(\mathrm{kV} / \mathrm{m})^{-1}\left(\mathrm{~g} / \mathrm{cm}^{2}\right)^{-1}, \\
\left.W_{n E}\left(h, h_{o}, R_{c}=10.8 \mathrm{GV}, m=\operatorname{tot} ; 1 ; 2 ; 3\right)\right|_{\mathrm{high} \mathrm{SA}} ^{\mathrm{Mt} \text { Hermon }} \\
\approx(4.72 ; 6.99 ; 4.66 ; 1.83) \times 10^{-5} \%(\mathrm{kV} / \mathrm{m})^{-1}\left(\mathrm{~g} / \mathrm{cm}^{2}\right)^{-1} .
\end{gathered}
$$

4. Observations of atmospheric electric field effects in total neutron intensity and in different multiplicities, and comparison with theory

\subsection{Measurements of AEF on Mt. Hermon; characteristics of thunderstorm periods}

The AEF sensor EFS-1000 started to work in the Emilio Segre' Observatory on Mt. Hermon in February 2000. It made measurements on top of the Observatory each minute for negative field up to $160 \mathrm{kV} / \mathrm{m}$ and for positive field up to $+16 \mathrm{kV} / \mathrm{m}$ (if the electric field has intensity $E>+16 \mathrm{kV} / \mathrm{m}$, EFS1000 shows only the upper limit $+16 \mathrm{kV} / \mathrm{m}$ ). In Figs. 1 and 2 are shown examples of these measurements.
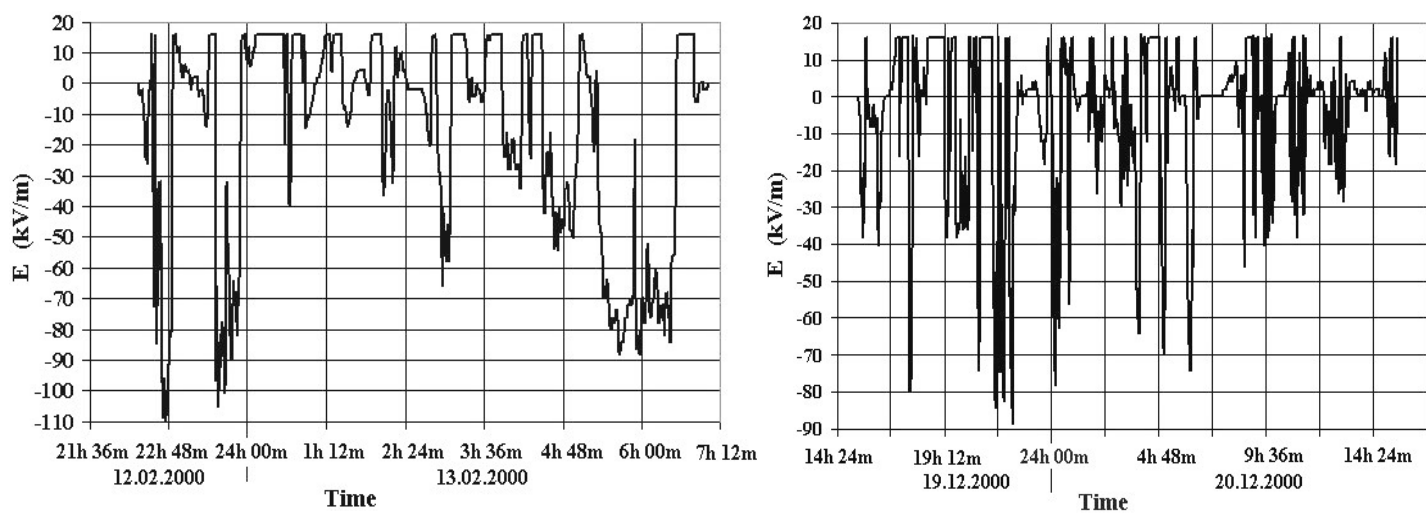

Fig. 1. One-minute data of AEF on Mt. Hermon during a thunderstorm periods in February 2000.

Fig. 2. The same as in Fig. 1, but for a thunderstorm periods in December 2000.

\subsection{One-minute data of atmospheric electric field and cosmic ray neutron intensity}

Because the expected AEF effect for measurements by NM on mountain heights is very small (see 
Section 3.4), it is necessary to decrease fluctuations in CR intensity caused by other causes than AEF effects. Therefore we corrected data of the total intensity and different multiplicities on barometric effect, on snow effect, and on primary variations (by using Rome NM data for comparison). Our one minute data are characterized with statistical errors of $0.98 \%, 1.45 \%, 2.76 \%$ and $4.74 \%$ for total intensity, $\mathrm{m}=1,2$, and 3 , respectively. Therefore we used averaged of many thousand one-minute thunderstorm EFS-1000 data.

\subsection{Regression relations between $\mathrm{AEF}$ and counting rates of total neutron intensity and different mul- tiplicities}

Unfortunately, the information on the space-time distribution of AEF E(h,t) is not available. In the Emilio Segre' Observatory on Mt. Hermon we have one minute data of continuous measurements only of $E\left(h_{O}, t\right)$ in the place occupied by Emilio Segre' Observatory. We suppose that $E\left(h_{O}, t\right)$ is in good correlation with distribution function $\mathrm{E}(\mathrm{h}, \mathrm{t})$. In this case we obtain approximately

$$
\left(\Delta I_{m}(t) / I_{m o}\right)_{E}=\int_{h_{3}}^{h_{o}} W_{m E}(h) E(h, t) d h \approx \bar{W}_{m E} \times\left(h_{O}-h_{3}\right) \times E\left(h_{o}, t\right),
$$

where $h_{3}$ is the air pressure on altitude of charged clouds caused thunderstorms.
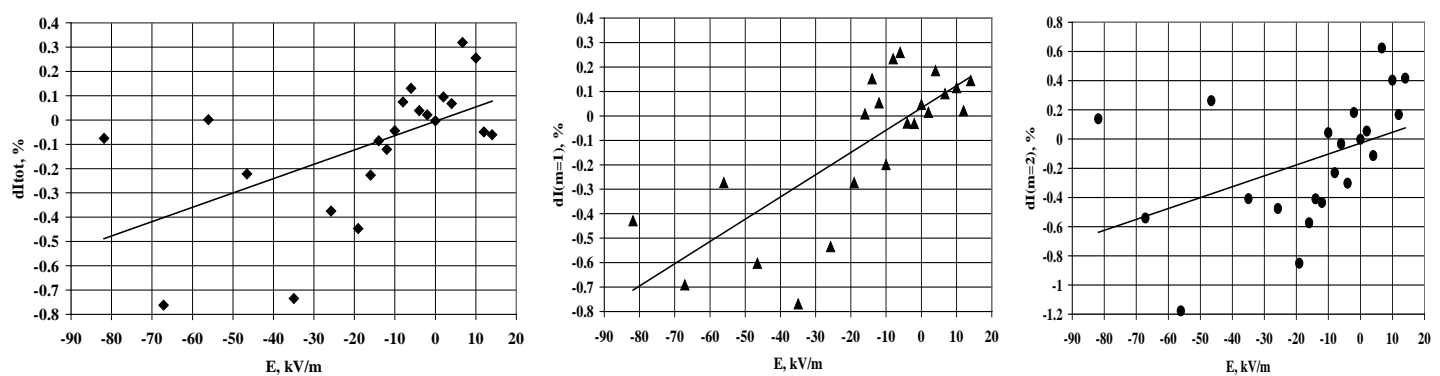

Fig. 3. CR intensity variations vs $\mathrm{E}$ for total neutron intensity, $\mathrm{m}=1$, and $\mathrm{m}=2$.

The statistical relation between variations of total neutron intensity and $\mathrm{E}$ is following:

$$
\left(\Delta I_{t}(t) / I_{t o}\right)_{E} \propto \bar{W}_{t E} \times\left(h_{o}-h_{3}\right) \times E\left(h_{o}, t\right)
$$

with correlation and regression coefficients

$$
R_{t}=0.56 \pm 0.09 ; \bar{W}_{t E} \times\left(h_{o}-h_{3}\right)=(0.0059 \pm 0.0018) \%(\mathrm{kV} / \mathrm{m})^{-1} \text {. }
$$

The statistical relations of multiplicities $\mathrm{m}=1,2,3$ with $\mathrm{E}\left(\mathrm{h}_{0}, \mathrm{t}\right)$ are as following:

$$
\left(\Delta I_{m}(t) / I_{m o}\right)_{E} \propto \bar{W}_{m E} \times\left(h_{o}-h_{3}\right) \times E\left(h_{o}, t\right)
$$

with correlation coefficients

$$
R_{1}=0.77 \pm 0.06, \quad R_{2}=0.45 \pm 0.11, \quad R_{3}=-0.07 \pm 0.14
$$

and regression coefficients

$$
\begin{aligned}
& \bar{W}_{1 E} \times\left(h_{O}-h_{3}\right)=(0.0091 \pm 0.0016) \%(\mathrm{kV} / \mathrm{m})^{-1}, \\
& \bar{W}_{2 E} \times\left(h_{O}-h_{3}\right)=(0.0075 \pm 0.0032) \%(\mathrm{kV} / \mathrm{m})^{-1}, \\
& \bar{W}_{3 E} \times\left(h_{O}-h_{3}\right)=-(0.0016 \pm 0.0048) \%(\mathrm{kV} / \mathrm{m})^{-1},
\end{aligned}
$$

4.4. Comparison of experimental results and theoretical predictions of AEF effects in NM total intensity and different multiplicities

The comparison of experimental results reflected in Fig. 3 and Eq. 4.2-4.8 shows that the best correlation with the highest regression coefficient was observed for the multiplicity $\mathrm{m}=1$. Lower correlation and smaller regression coefficients were observed for the total neutron intensity and for multiplicity $m=2$. Practically null correlation and negligible regression coefficient were observed for the multiplicity $m=3$. These results are in good qualitative agreement with those predicted by the theory of Dorman and Dorman (1995), Dorman et al. (1995), Dorman and Dorman (1999) that was described above in Section 4.3.

Quantitative comparison of experimental results with theory is much more difficult. On the one hand, the influence of AEF on charged energetic particles of CR has an integral character in vertical direction from the level of observations to the level of charged clouds and from great horizontal surface (neutron monitor detects particles arrived mostly from zenith angles smaller than about $30^{\circ}$ which means from the surface about $10 \mathrm{~km}^{2}$ on the height of charged clouds of few $\mathrm{km}$ ). On the other hand, the sensor of AEF 
EFS-1000 gives information only on the local AEF on the top of our Observatory on Mt. Hermon. Nevertheless we can try to do this comparison and estimate approximately the average depth (in $\mathrm{g} / \mathrm{cm}^{2}$ ) of layer where CR particles are accelerated and decelerated. From results, obtained for $\mathrm{m}=1$ we obtain

$$
\left(h_{o}-h_{3}\right)_{m=1} \approx \frac{(0.0091 \pm 0.0016) \%(\mathrm{kV} / \mathrm{m})^{-1}}{6.99 \times 10^{-5} \%(\mathrm{kV} / \mathrm{m})^{-1}\left(\mathrm{~g} / \mathrm{cm}^{2}\right)^{-1}}=(130 \pm 23) \mathrm{g} / \mathrm{cm}^{2}
$$

For total intensity and multiplicity $\mathrm{m}=2$ we obtain

$$
\begin{aligned}
& \left(h_{o}-h_{3}\right)_{t o t} \approx \frac{(0.0059 \pm 0.0018) \%(\mathrm{kV} / \mathrm{m})^{-1}}{4.72 \times 10^{-5} \%(\mathrm{kV} / \mathrm{m})^{-1}\left(\mathrm{~g} / \mathrm{cm}^{2}\right)^{-1}}=(125 \pm 38) \mathrm{g} / \mathrm{cm}^{2} \\
& \left(h_{o}-h_{3}\right)_{m=2} \approx \frac{(0.0075 \pm 0.0032) \%(\mathrm{kV} / \mathrm{m})^{-1}}{4.66 \times 10^{-5} \%(\mathrm{kV} / \mathrm{m})^{-1}\left(\mathrm{~g} / \mathrm{cm}^{2}\right)^{-1}}=(160 \pm 68) \mathrm{g} / \mathrm{cm}^{2}
\end{aligned}
$$

According to Eq. 4.8 AEF effects for $\mathrm{m}=3$ and higher are negligable (in agreement with considered theory) and it is no sense to take them into account. On the basis of Eqs. $4.9-4.11$ we obtain final average result

$$
\left(h_{o}-h_{3}\right)_{a v}=(132 \pm 19) \mathrm{g} / \mathrm{cm}^{2},
$$

which shows that charged clouds are in average on about 3/2 km over Mt. Hermon what not contradict meteorological observations.

Acknouladgements: L.D. and L.P. cordially thank Ministry of Science of State Israel, Tel Aviv University, the 3-rd Rome University, Pushkov's IZMIRAN of Russian Academy of Science, Shamir Research Institute, and Israel Space Agensy for continue supporting of the work of Emilio Segre' Observatory on Mt. Hermon

\section{References}

Alexeenko V.V., A.E. Chudakov, V.G. Sborshikov, and V.A. Tizengauzen "Short perturbations of cosmic ray intensity and electric field in atmosphere”, Proc. 19th Intern. Cosmic Ray Conf., La Jolla, 5, 352355 (1985).

Alexeenko V.V., A.B. Chernyaev, A.E. Chudakov, N.S. Khaerdinov, S. Kh. Ozrokov, and V.G. Sborshikov "Short perturbations of cosmic ray intensity and electric field in atmosphere", Proc. 20th Intern. Cosmic Ray Conf., Moscow, 4, 272-275 (1987).

Dorman L.I. "To the theory of cosmic ray meteorological effects", Doklady Academy of Sciences of USSR (Moscow), 94, No. 3, 433-436 (1954).

Dorman L.I., Cosmic Ray Variations. Gostekhteorizdat, Moscow, M1957. (English translation: US Department of Defense, Ohio Air-Force Base, 1958).

Dorman L.I., Meteorological Effects of Cosmic Rays. NAUKA, Moscow, M1972

Dorman L.I. and I.V. Dorman "Cosmic-ray atmospheric electric field effects", Canadian J. of Physics, 73, 440-443 (1995).

Dorman L.I. and I.V. Dorman "Formation of lead mesoatoms in neutron monitor by soft negative muons and expected atmospheric electric field effect in the cosmic ray neutron component”, Proc. 26th Intern. Cosmic Ray Conf., Salt Lake City, 7, 309-312 (1999).

Dorman L.I., I.V. Dorman, N. Iucci, M. Parisi, G. Villoresi, and I.G. Zukerman 'Emilio Segre' Observatory and expected time-variations in neutron monitor total and multiplicities counting rates caused by cosmic ray particle energy change in the periods of thunderstorms”, Proc. 26th Intern. Cosmic Ray Conf., Salt Lake City, 7, 425-428 (1999).

36th International Cosmic Ray Conference -ICRC2019-

July 24th - August 1st, 2019

Madison, WI, U.S. 
Title (or short title)

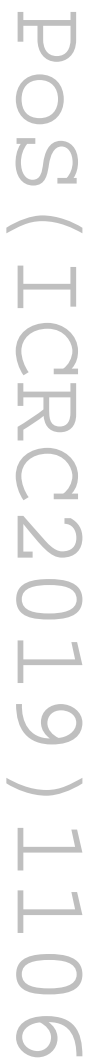

